\title{
Integrating Implicit Structure Visualization with Authoring Promotes Ideation
}

\author{
Andrew M. Webb and Andruid Kerne \\ Interface Ecology Lab \\ Dept. of Computer Science \& Engineering, Texas A\&M University \\ \{andrew, andruid\}@ecologylab.net
}

\begin{abstract}
We need to harness the growing wealth of information in digital libraries to support intellectual work involving creative and exploratory processes. Prior research on hypertext authoring shifted the focus from explicit structure to direct presentation of content aided by "implicit" spatial representation of structure. We likewise shift the field of information visualization. Using hypertext's rubric, we redefine what most people think of as "information visualization" as explicit structure visualization. We alternatively address implicit structure visualization, presenting content directly, representing structure with spatiality and other visual features. We integrate authoring to emphasize the role of human thought in learning and ideation. Prior research has shown that people iteratively collect and organize information by clipping magazines, piling clippings in somewhat messy ways, and organizing them.

MessyOrganizer is an iterative implicit structure visualization algorithm which, like human practice, gradually collects and organizes information clippings. Content is depicted directly. Structural relationships are visualized implicitly through spatial positioning of related elements, with overlap and translucence. The simulated annealing algorithm is applied to a model of semantic relatedness over a spatial grid. We develop an experiment comparing products created with the integrated environment versus separated visualization and authoring spaces. Results reveal that participants have more novel and varied ideas when visualization is integrated with authoring.
\end{abstract}

\section{Categories and Subject Descriptors}

H.5.m [Information Systems]: Information Interfaces and Presentation-miscellaneous.

\section{General Terms}

Algorithms, Design, Experimentation, Human Factors

\section{Keywords}

creativity support tools, information visualization, authoring

\section{INTRODUCTION}

The increasing abundance of information in digital libraries can

Permission to make digital or hard copies of all or part of this work for personal or classroom use is granted without fee provided that copies are not made or distributed for profit or commercial advantage and that copies bear this notice and the full citation on the first page. To copy otherwise, or republish, to post on servers or to redistribute to lists, requires prior specific permission and/or a fee.

JCDL'11, June 13-17, 2011, Ottawa, Ontario, Canada

Copyright 2011 ACM 978-1-4503-0744-4/11/06 ..\$10.00. support creative processes for humans engaged in intellectual work like design, writing, learning, and problem solving. Consider a scenario in which a hypothetical high school student, Grace, uses Wikipedia to research ideas for a science project. Grace will explore a wide space of ideas across many documents. She will seek to understand relationships between the documents, collect and organize relevant information, annotate thoughts, think about the information, and develop a research topic. She could make use of explicit structure information visualizations, such as treemaps [43] or node-link diagrams [33] to understand relationships. However, content would not be directly visible.

In intellectual work involving creativity, learning, or problem solving, content is at least as important as relationships between elements. For example, knowing when people posted images about Hurricane Katrina to Flickr, and with what tags, is useful, but seeing the images has another value [13]. Visualizing content and explicit structure together increases visual complexity, disrupting legibility. Implicit structure visualization expresses relationships through visual features such as spatial positioning, size, and translucence without adding new visual elements (see Figure 1). As prior information visualization uses explicit structure to convey relationships in abstract data to amplify cognition [7], we find that implicit structure form of information visualization represents relationships between information concepts to amplify sensemaking and ideation. Implicit structure visualization seeks to support users in simultaneously thinking about information content and understanding relationships while avoiding too high a demand on visual attention.

As part of intellectual work, Grace engages in exploratory search, actively investigating information to learn something new [22]. The goal is to learn. Information-based ideation is the creative process where new ideas emerge from seeing relationships between existing ideas while working with information [19]. This can occur when Grace is authoring a prior work collection for her science project, highlighting a new focus for her research. Information-based ideation tasks involve assembling and seeing relationships among collected information, developing new ideas, and connecting answers to open-ended questions [20].

In exploratory search and information-based ideation tasks, people author collections with annotations to help them learn and formulate new knowledge. In building her prior work collection, Grace not only collects relevant documents. She annotates ideas on how research topics relate to each other. She organizes the information in a meaningful way. Authoring is essential to information-based ideation [18]. New ideas emerge for Grace when previously unrelated elements are juxtaposed (Figure 1).

Authoring is typically separated from visualization. Grace would drag and drop information from the visualization to a different 


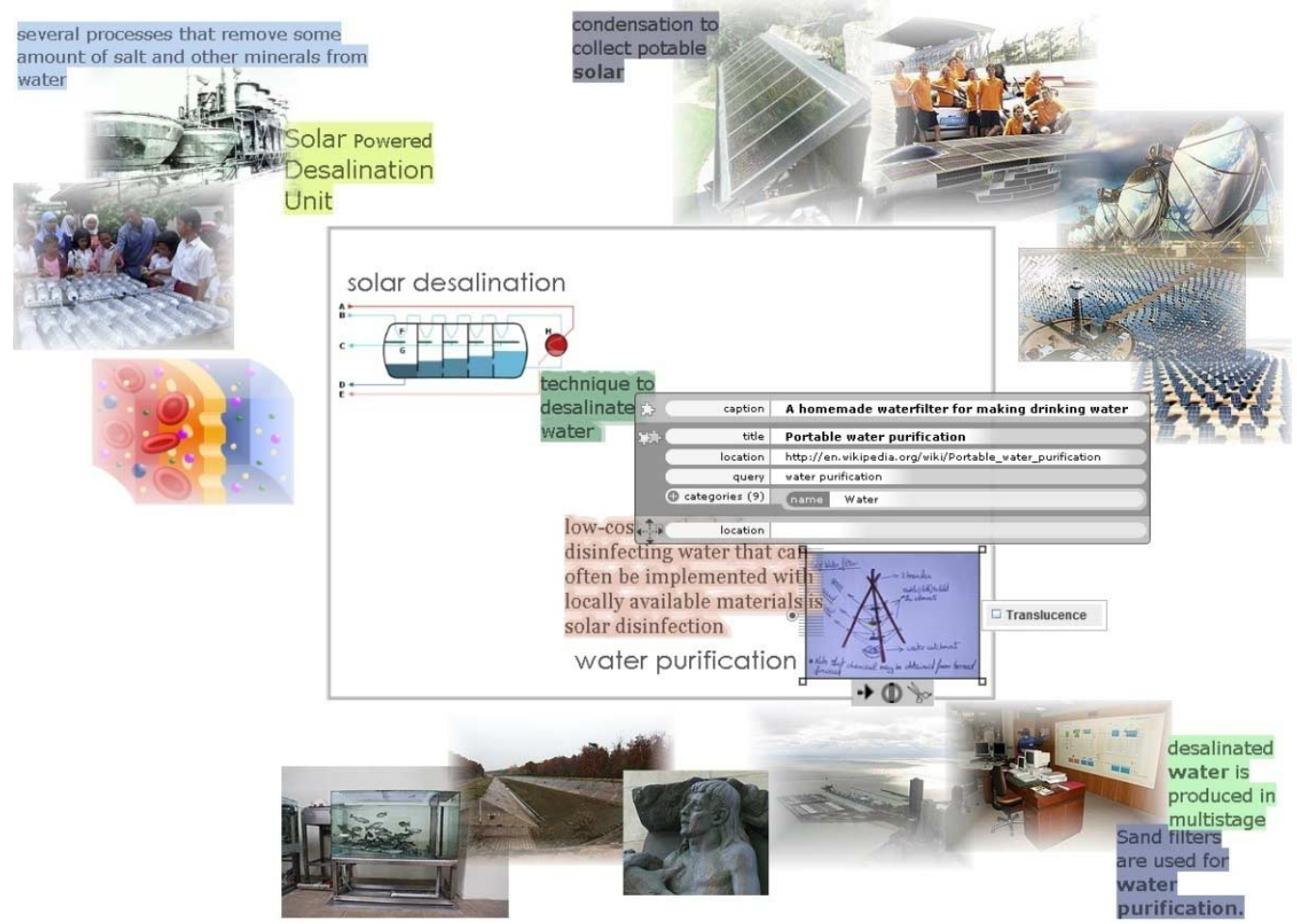

Figure 1: Snapshot of an implicit structure visualization integrated with authoring being used by Grace to develop ideas for her science project, a low cost solar powered salt water purifier. In the center, she is authoring a collection of surrogates. In-context metadata details-on-demand for one surrogate are displayed. In the peripherae, MessyOrganizer visualizes extracted surrogates.

application for authoring. Effort increases as she must switch context from exploring to authoring. Visual elements change, increasing demand on attention. Information semantics are lost in the transfer. We hypothesize that integrating implicit structure visualization with authoring will support information-based ideation. We developed MessyOrganizer, a new implicit structure visualization algorithm that supports integration with authoring. In this integration, the user is not only modifying parameters of the visualization, but changing the visualization through direct manipulation. The user rearranges elements, changing implicit structure, and connects processes of collecting and developing ideas. We will address design challenges arising from how integration affects ideation, visual representation, and interaction.

We begin with a discussion of background material. We then explain the integration of implicit structure visualization with authoring. Next, we detail MessyOrganizer, our new incremental implicit structure visualization algorithm, and how it integrates with authoring. We evaluate the effect of integrating implicit structure visualization with authoring on ideation. We conclude by discussing implications for design of integrated environments.

\section{BACKGROUND}

This research is grounded in creative cognition. Prior work in the field develops evaluation methods for ideation tasks. We examine annotation, a subset of authoring that is important to ideation and learning. Implicit structure relates directly to authoring and our approach to visualization. We draw on related work and explain an experience gap within information visualization. We explore explicit structure information visualization in digital libraries. We use clippings to represent content as surrogates. The integration of implicit structure visualization with authoring is investigated through mixed-initiative information composition.

\subsection{Creative Cognition}

The creative cognition approach is grounded in the perspective that creativity results from a federation of cognitive mechanisms [38]. Creative ideation means having ideas. Ideas arise during cognitive tasks. Ideation is difficult to measure. Cognitive tasks involve either convergent or divergent thinking. Performance of convergent thinking tasks requires a single correct answer. Results are measured by speed and accuracy. These tasks and measures, which are widely used, are insufficient for creative cognition [12] and important areas of research into interactivity and information [4]. Divergent thinking tasks, in which one quests for many possible answers to open-ended questions, are designed to investigate creative ideation. Exploratory search and informationbased ideation tasks both involve divergent thinking.

Shah, Smith et al. developed creative ideation metrics to evaluate performance of divergent thinking tasks: quantity, quality, novelty and variety of ideas [32]. Kerne et al extended these metrics for tasks involving information-based ideation and discovery [20].

Quantity of Ideas is assessed through evaluators breaking concepts down into individual functional solutions and then counting them. Having more ideas is important in Darwinian models of creativity, as more possible solutions are considered [36].

Quality is a measure of a product solution's feasibility, clarity, and how well it meets design specifications.

Novelty measures how unusual a product solution is. The concepts are sorted into bins of similar or identical solutions. We count how many experiment participants developed each.

Variety measures the span of categories in an answer.

\subsection{Annotations}

In information-based ideation tasks, annotations are used to 
express novel ideas. Annotations have a role in supporting intellectual work. Marshall found that college students use annotations in textbooks as procedural signals, place markings, in situ problem working, and interpretive activity [23]. Annotating while reading supports understanding [27]. People annotate web documents to aid navigation and organization [14].

\subsection{Authoring Implicit Structure}

We consider the role of structure in authoring and information visualization experiences. Beginning with Aquanet, Marshall and Rogers saw hypertext authors engaging in sensemaking tasks [30] eschew formal representations of semantics, instead opting for spontaneous organization [25]. This finding transformed hypertext research, moving from reasoning systems (e.g. gIBIS [9]) to spatial hypertext [26], and Kerne et al's work in parallel, on more visual information composition [18]. Information composition is similar to spatial hypertext in terms of information layout, but differs in emphasis on visual design and communication.

\subsection{Visualization of Implicit Structure}

"Formality Considered Harmful" retrospectively examines how converting thinking processes into formal models, such as nodelink structures, and schematized types, imposes extra cognitive steps into sensemaking, diminishing usability [34]. We apply this analysis to examine the roles of formal and informal structure in information visualization. Information visualization, e.g. with ManyEyes [40] or NodeXL [37], typically requires such formalization. Many document semantics visualizations focus on structural features like author relationships [e.g., 18]. It is difficult to include the information content itself, such as images clipped from documents, because they are visually complex and heterogeneous.

The assumption of explicit structure representation can lead to an information visualization experience gap. Amar and Stasko identified gaps between representation and analysis [3]. Creative cognition is a super-set of analysis, exacerbating the problem. In response, we emphasize the representation of meaning. Simply visualizing structures is not sufficient for many sensemaking, problem solving, and ideation tasks, because the user may be very interested in the content, in the ideas inherent in each element. Our approach makes presentation of content, with image and text clippings, primary, while presenting relationships with implicit structure visualization. Just as what became spatial hypertext would not have been seen as hypertext in the field's early days, so the MessyOrganizer implicit structure visualization is a new form of information visualization, with a different look than that developed by many prior practitioners.

\subsection{Digital Library Information Visualization}

Several researchers have investigated visualizations for digital libraries and information collections, but none to our knowledge have addressed integrating authoring with the visualization. Xu et al designed and evaluated a treemap visualization for personal media collections [43]. Butcher et al explored search interfaces for digital libraries using multimedia strand maps, which visualize connections between concepts through explicit graph edges, to support conceptual discovery [6]. Druin et al developed SearchKid, a visual interface for searching, browsing, and viewing results for a children's digital library [10]. They found that children wanted to use information they found, so they supported collection authoring, but through a separate application. Shen et al formalize the integration of browsing, searching, and visualization in digital libraries as theory [33]. They suggest blurring the line between searching and browsing to support exploratory search.
We take this one step further, integrating authoring to promote thinking about the information in creative ways. World Explorer uses implicit structure visualization to show aggregated data from Flickr images as variable sized tags spatially overlaid on a map [2]. Card et al used real world metaphors as a basis for WebBook, a visualization for grouped web pages in virtual books, and Web Forager, a 3D information workspace with virtual bookshelves [8]. Robertson et al developed Data Mountain to aid document management by visualizing documents on an inclined plane in a $3 \mathrm{D}$ virtual environment where users can arrange and organize, supporting authoring implicit structure [29].

\subsection{Surrogates}

How documents are represented in a collection impacts user experience. A surrogate is a representation of an information resource that provides access to that resource [5]. Wildemuth et al investigated fast-forward surrogates for representing video documents within digital libraries [42]. Green et al examined how previews and overviews can be used as surrogates to support exploratory search [15]. A preview is a single image thumbnail, text clipping, or analytic object (e.g. bar in graph). Overviews combine visual objects to convey relationships. In mixed-initiative information composition, informative documents are represented by image and text surrogates that function as clippings, visualizing content and supporting implicit structure visualization [18]. Information composition surrogates provide navigation to textual documents to support exploration of concepts in intellectual work.

\subsection{Mixed-initiative Information Composition}

To investigate how integrating implicit structure visualization with authoring can promote information-based ideation, we develop mixed-initiative information composition [18]. Composition, a term that comes from the arts, means an assemblage of connected elements to form a whole. Composition uses implicit structure to visualize relationships. In Grace's composition, she spatially arranges surrogates in groups, such as solar panels, water purification and desalination. She emphasizes importance of ideas through surrogate sizes. Users create information compositions to answer open-ended divergent thinking questions, such as developing a research topic or planning a family vacation. It has been shown that authoring information compositions stimulates emergence of new ideas on information-based ideation tasks [20].

combinFormation is a creativity support tool that uses mixedinitiative information composition to represent information collections [18]. In combinFormation, computational agents extract image and text surrogates from documents and visualize them to the user in a composition space. The human user collects and organizes surrogates and annotates ideas to author a composition to answer open-ended question as part of an information-based ideation task.

The prior visualization algorithm in combinFormation used a spreading activation technique [28][18] that placed new surrogates by giving high activation to unoccupied regions near the most relevant surrogates. This approach begins to fail as the visualization space fills with surrogates. The spread radius only extends so far from a surrogate. Once a surrogate is surrounded, future placements of related surrogates become random as no regions have adequate activation.

\section{INTEGRATING IMPLICIT STRUCTURE VISUALIZATION WITH AUTHORING}

Integrating implicit structure visualization with authoring connects human processes for exploring and creating while working with 
information. Prior environments support either exploratory processes through explicit structure visualization or creative processes through collection authoring and annotation. Hypertext browsing enables exploration of content. We hypothesize that connecting these processes will support users engaged in information-based ideation. Here we articulate issues associated with integration: connected visual spaces, serendipitous promotion of ideation, underlying semantics in the user experience, and interactive tools.

Since monitors and the human field of vision are limited, visual space is at a premium. We emphasize human ideation as central to using information collections in intellectual work. To use space effectively, we divide the composition space into two regions, one in the center and the other peripherae (see Figure 1). The primary authoring space, which we call the cool space is located at the center. Changes in this space are only made by the user.

Implicit structure visualization is generated in a peripheral region, called the hot space. MessyOrganizer is used to add and remove surrogates here. The cool space leaves a hole in the hot visualization space. Unlike prior visualizations, which occupy a contiguous rectangle [43], this constraint adds new complexity to the visualization algorithm. While the composition space is divided, the human process of authoring is integrally supported in both spaces. Grace is not limited to annotating or collecting information in only the cool space. She can also use the hot space to collect and annotate information for future reference.

Visualization in the peripherae serves to aid creative processes. Engaging surrogates serendipitously appear, stimulating new insights. For example, as Grace authors ideas for her science project, an interesting surrogate of a portable water purifier appears, leading her to expand her research scope to address portability.

An advantage of integration is that collection elements do not lose information semantics when moving between visualization and authoring spaces. Metadata, such as the author of a publication, caption for an image, and title and category for a Wikipedia article, is retained, providing context to support sensemaking. Semantics can influence visualization, such as algorithmic placement of surrogates in the hot space near related surrogates collected by the user in the cool space.

Interactive tools support visualization and authoring across the spaces. The user affects information collection and visualization through an in-context interface for expressing importance of semantic features [41]. The user also adjusts visual features, such as spatial positioning, size, color, and translucence. She can group related surrogates and connect relationships between existing ideas, supporting information-based ideation. She can adjust the proportions of the cool and hot spaces, when authoring or visualization generation is more important in her task. In information-based ideation, a person alternates exploratory and creative processes, from browsing and collecting information to authoring ideas and vice versa [17]

\section{MESSYORGANIZER VISUALIZATION}

We developed MessyOrganizer, a new incremental implicit structure visualization algorithm that supports integration with authoring. The implicit structure and incremental steps mimic human processes of collecting and organizing clippings. Emerging collections inevitably grow messy, because we lack a complete $a$ priori cognitive schema. The resulting loosely organized messiness helps the user think about the information and encourages rearrangement. In this section, we motivate and develop this algorithmic messiness. We describe how MessyOrganizer uses implicit structure visualization to layout surrogates incrementally based on functions designed to minimize messiness, and manage the mess by removing older surrogates.

\subsection{Clippings and Messiness}

Marshall and Bly found that people desire organization of physical clippings to avoid appearing a "pack rat" [24]. Participants organized related clippings by pinning them next to each other on a wall. Clippings overlapped. Older clippings became buried underneath newer ones. MessyOrganizer simulates this collection process by spatially grouping and overlapping related surrogates.

MessyDesk, inspired by human practices of organizing information on physical desks through messy piles, was designed to help people organize and remember information by enticing them to develop context through decoration of a desktop space [11]. MessyOrganizer extends the role of messy organization used in MessyDesk to support automated grouping of similar elements through use of white space, overlap, and translucence. In MessyDesk, users arrange information in spatial groups to help organize information. Groupings are the basis for emergent ideas in information-based ideation [20] and chunking [35].

The practice of mixed-initiative information composition, by students in a course on invention and entrepreneurship, produced corroborative evidence [17]: "I like things in an order. Through the process of making messy things organized, I came up with new ideas. If everything is linear and in order, there is no need to think anything new." Messiness was found to play a formative role in creative cognition and situated creative learning.

\subsection{Visualization Algorithm}

We simulate iterative human processes of collecting and organizing clippings to support creative cognition in informationbased ideation tasks. A person does not know in advance exactly what she will collect or how to organize it. Concepts and associated structures emerge. Thus, things get out of place as we visualize new information. While building personal collections, significance of ideas and focus on concepts shift. MessyOrganizer is designed to support these shifts. The messiness of MessyOrganizer engages users in organization of information.

MessyOrganizer is an incremental visualization algorithm, designed to operate on a dynamically changing collection. We define incremental visualization formally as a dynamic presentation created by a sequence of placement decisions. Each decision places only a single element. Its operation is independent of the previous decision, as each placement runs separately. However, each placement changes the state of the visualization as a whole, and each placement decision is based on that whole state. Thus, the range of possible visualizations at any time $\mathrm{n}$ is constrained by the visualization at time n-1, making global optimization without moving elements impossible.

We derive the MessyOrganizer algorithm from simulated annealing. Simulated annealing is a stochastic algorithm that models the annealing process in metallurgy to search a problem space to find an optimal solution [21]. In annealing, a metal is repeatedly heated to a high temperature, then cooled slowly, resulting in a restructuring of the crystallization and improving the metal's durability. In a similar manner, MessyOrganizer attempts to find an optimal visualization (least messy) by minimizing an energy function for each element placed. The minimization of the energy function is performed by iteratively adjusting a potential 


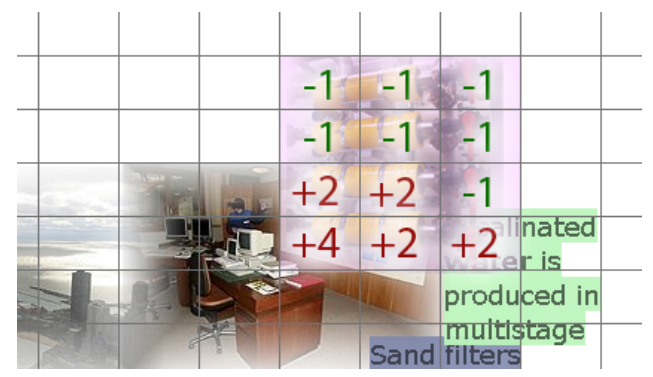

Figure 2: Example of MessyOrganizer attempted placement for an image. Fat pixel grid lines are displayed. Effects on energy function associated with each fat pixel are displayed.

placement in small amounts, until the energy function is less than the desired value or until number of iterations exceeds a threshold. If the energy requirement is met, the placement is successful, and the element is placed. Otherwise, time expires, and the placement fails. A transition function uses a decaying temperature equation to simulate the cooling process affecting when a placement adjustment is accepted, allowing more changes in early iterations and fewer in later ones. Moves from lower to higher energy are allowed during earlier iterations to avoid local minima.

\subsection{Layout: structuring the messiness}

In MessyDesk, a user lays out information in groups and piles to develop meaningful contexts to help in organization and remembering. MessyOrganizer is designed to automatically visualize meaningful contexts by grouping related surrogates and minimizing distance between related information. The goal of the algorithm is to generate the optimal layout of surrogates by minimizing the mess created with each new surrogate.

As a basis for layout, the integrated visualization and authoring space is divided into a grid of fat pixels (see Figure 2). A fat pixel is a rectangular area consisting of multiple pixels and having the same aspect ratio as the whole visual space. We define placement of a surrogate by the fat pixel that will contain the surrogate's top left corner. A set of iterative placements constitutes a layout. MessyOrganizer selects an initial placement to begin calculating optimal placement for a new surrogate. We determine the initial placement by calculating an activation weight for each fat pixel based on the similarity between the surrogates in that fat pixel and the surrogate being placed. The similarity function uses semantic features in the form of term vectors [31]. This algorithm could also sensibly be used with a similarity function based on visual features, or a combination of both. Fat pixels containing surrogates more related to the surrogate being placed will have higher weights. The fat pixel with the highest weight is selected for initial placement. If the highest weight is shared by more than one fat pixel, the average weight of the fat pixels surrounding each of the highest weight fat pixels is calculated. The fat pixel with the highest average is selected for initial placement.

As a result of the environment's integration, information authored by the user in the cool space also impacts visualization. Activation is spread from fat pixels in the cool space across the border to the hot space. The result is increased activation for fat pixels in the hot space that are near related surrogates in the cool space. The goal is to help the user more easily make sense of the new information by placing it near already collected information. This helps maintain visual groupings so that information can be chunked effectively.

\subsection{Functions: minimizing the messiness}

When people organize physical clippings or digital information with MessyDesk, the messy organization has meaning. MessyOrganizer aims to develop meaningful messiness through the following functions for simulated annealing algorithms: (1) an energy function to evaluate messiness of a placement, (2) a neighbor function to make small changes to messiness, and (3) a transition function to navigate the messiness. The energy function calculates the quality of the current placement. The goal of MessyOrganizer is to minimize the energy function. The neighbor function specifies a new placement based on a small change in the existing placement. The transition function specifies probability of transitioning to a new placement based on the energy for both the current and new placements, and temperature reflected by a percentage of time.

\subsubsection{Energy Function}

MessyOrganizer spatially groups related surrogates, using overlap along edges to connect groups. This is reflected in the energy function. The energy function for MessyOrganizer is presented in Equation 1, where $C$ represents a constant, $x$ and $y$ represent the fat pixel grid coordinates of the top left corner for surrogate placement, $w$ and $h$ are the width and height in fat pixels of the surrogate, and $d$ is the distance to the nearest fat pixel containing the most related surrogate.

$$
\begin{gathered}
\text { energy }(x, y, w, h, d)=C_{d} \cdot d+\frac{1}{w h} \sum_{i=x, j=y}^{i=x+w, j=y+h} f(i, j) \\
f(i, j)=\left\{\begin{array}{cl}
C_{\text {edge }}, & \text { if fat pixel contains edge of surrogate } \\
C_{\text {middle }}, & \text { if fat pixel contains middle of surrogate } \\
C_{\text {empty }}, & \text { if fat pixel is empty }
\end{array}\right. \\
\text { where, } C_{\text {edge }}<C_{\text {middle }} \text { and } C_{\text {empty }}<0
\end{gathered}
$$

Messiness increases when related surrogates are not spatially near each other. Thus, energy increases the further away (in Euclidean distance) a surrogate is from the most related surrogate. Messiness is not only about spatial grouping. Occlusion of information also results in meaningless messiness. Energy increases when a placement contains a fat pixel that already has surrogates. Energy decreases for each empty fat pixel a new surrogate would occupy. These fat pixel based energy changes are dependent on $f(\mathrm{i}, \mathrm{j})$ which returns a positive value when a fat pixel already has a surrogate and a negative value when it is empty. A smaller positive value is returned when a surrogate edge occupies the fat pixel. The result allows overlap between surrogates, but only along surrogate edges. Surrogates come in different sizes and occupy varying numbers of fat pixels. The average of the energy changes resulting from $f(\mathrm{i}, \mathrm{j})$ is used to avoid advantages for larger surrogates. As mentioned above, integrating visualization with authoring requires that layout avoids using the central cool subspace allocated only for authoring. Any placement that results in part of a surrogate lying in the cool space or outside the composition space receives an extremely high energy to prevent success of such placements. Figure 2 shows an example of an attempted placement with fat pixels labeled with energy values. This placement will fail because of too much overlap with existing surrogates.

The constants serve as weights impacting the effect of components of the energy function. $\mathrm{C}_{\mathrm{d}}$ affects the importance of distance. A higher value will require placements closer to the most related surrogate. $\mathrm{C}_{\text {edge }}$ and $\mathrm{C}_{\text {middle }}$ affect the importance of overlap. By making $\mathrm{C}_{\text {edge }}$ less than $\mathrm{C}_{\text {middle }}$, overlap of surrogate edges will occur more frequently. If these two constants have the same value, overlap is not differentiated between the edge or middle of a surrogate. $\mathrm{C}_{\text {empty }}$ affects the importance of empty fat pixels. A 
higher negative value will require fewer empty fat pixels for placement. Adjusting these constants can achieve different effects depending on visualization goals.

\subsubsection{Neighbor Function}

Messiness can be adjusted by choosing a neighboring fat pixel. The neighbor function slightly adjusts placement. The function is given a fat pixel and returns a neighboring fat pixel for which placement has not been attempted. The function begins by returning those fat pixels directly adjacent and moves radially around the fat pixel increasing the radius by one after each full circumvention. The starting adjacent fat pixel is the one with most open space extending out in its direction which is determined by the location of the fat pixel in the composition space. For example, the first neighbor for a fat pixel located towards the top left corner of the composition space will be the lower right adjacent fat pixel.

\subsubsection{Transition Function}

In minimizing the messiness, we sometimes need to consider more messy placements in order to continue searching for the global minimum. The transition function allows more moves to messier placements in earlier iterations, and less in later iterations as we focus in on the optimal messiness. The transition function is defined as follows where $e_{c}$ is the energy for the current placement, $e_{n}$ is the energy for the new placement, $t$ is the temperature, and $k_{B}$ is the Boltzmann constant:

$$
T\left(e_{c}, e_{n}, t\right)=\left\{\begin{aligned}
1, & e_{n}<e_{c} \\
0, & e_{n}=e_{c} \\
\exp ^{\frac{e_{c}-e_{n}}{k_{B} \cdot t}}, & \text { otherwise }
\end{aligned}\right.
$$

Temperature change is a linear decay based on the percentage of the total number of interactions run, highest on the first iteration.

\subsection{Removing: managing the messiness}

Placement in MessyOrganizer fails when no solution with acceptable energy is found after a number of iterations. This failure occurs when there is not enough space to place a new surrogate. In incremental visualization, the collection is normally too large to be entirely placed in a given space. Techniques are needed to manage the messiness as new elements are added. Factors affecting visualization of heterogeneous information elements dictate how space is made available.

To make presentations of complex information intelligible, white space is required [39]. Without white space, groupings of semantically related information become impossible to recognize. At the extreme, having no white space at all will make recognition of individual elements extremely difficult. MessyOrganizer manages messiness by maintaining a ratio of positive to negative space. The algorithm is not allowed to place a new surrogate if the positive-negative ratio is too large. Instead, old surrogates are iteratively removed to meet the ratio requirement.

Determining which surrogates to remove is a challenging problem. In order to maintain spatial grouping of related surrogates, clearing space near other related surrogates is the ideal solution. However, this is not always possible. The ideal surrogate to remove may have just been added to the hot space. Surrogates need time for the user to see them. Removing a surrogate just as it was added is disruptive to the user and not beneficial to information-based ideation. Space made available might be too small for the new surrogate, requiring multiple surrogates to be removed. MessyOrganizer removes the oldest surrogates that are nearest in Euclidean distance to the most related surrogate(s).
At each placement, an aging process is applied to all surrogates in the hot space. This aging process desaturates images and increases the alpha translucence of text. The result is layers of separation [39] between surrogates based on time, increasing visibility of newer surrogates. The layering supports visualization of messy piles, which through integration with authoring can be reorganized and labeled by the user.

\section{EXPERIMENT}

We designed an experiment to validate our hypothesis, that integrating implicit structure visualization with authoring promotes creativity in information-based ideation tasks. Visualization algorithm and authoring tools are removed as experimental factors by using MessyOrganizer and information composition across conditions. We extend ideation metrics from creative cognition to evaluate the products.

\subsection{Experiment Design}

We conducted a 2 × 2 within-subjects experiment. Participants created information compositions while performing informationbased ideation tasks. Independent variables we manipulated were connectivity between visualization and authoring spaces (separated spaces vs. integrated spaces) and information-based ideation question asked. In separated spaces, the control condition, visualization of new surrogates by agents was performed in a separate window from the authoring space. Participants could not perform authoring operations in the separate visualization space. The only connectivity between the two spaces was through the drag and drop of surrogates from the visualization space to the authoring space. The control condition simulates the typical prior conditions for visualization and authoring of personal, task-oriented collections. Information visualization is conducted in one application, with browsing and searching [15]. Collecting and authoring are independently conducted by the user, using an application such as Microsoft Word.

The experimental conditions were divided into two phases. In the first phase, participants spent 18 minutes collecting visualized information and developing ideas. MessyOrganizer was used in both conditions to visualize new surrogates. In the separated condition, the participant collected information by dragging and dropping surrogates across windows from the visualization space to the authoring space. In the integrated condition, participants collected information by dragging and dropping surrogates from the hot space to the cool space. In the second phase, participants spent 10 minutes organizing, designing, and annotating to author an information composition. The visualization stopped in both conditions. No new surrogates would appear.

While a typical user can issue any combination of search queries she wants in combinFormation, to reduce variability in a controlled experiment, we pre-formulated the search queries for seeding information agents. We cached search results from Google on our own server, to eliminate variability in the ranking of results for each query.

There were 49 participants in the experiment. They were undergraduate introductory psychology students who fulfill a course requirement by either writing a paper or serving as subjects.

The experiment took place in two sessions. The first was a training session. Participants watched a 12-minute instructional video about information composition using combinFormation and answering information-based ideation questions. The video informed how compositions would be rated. Participants trained with both condition apparatuses. They answered two information- 


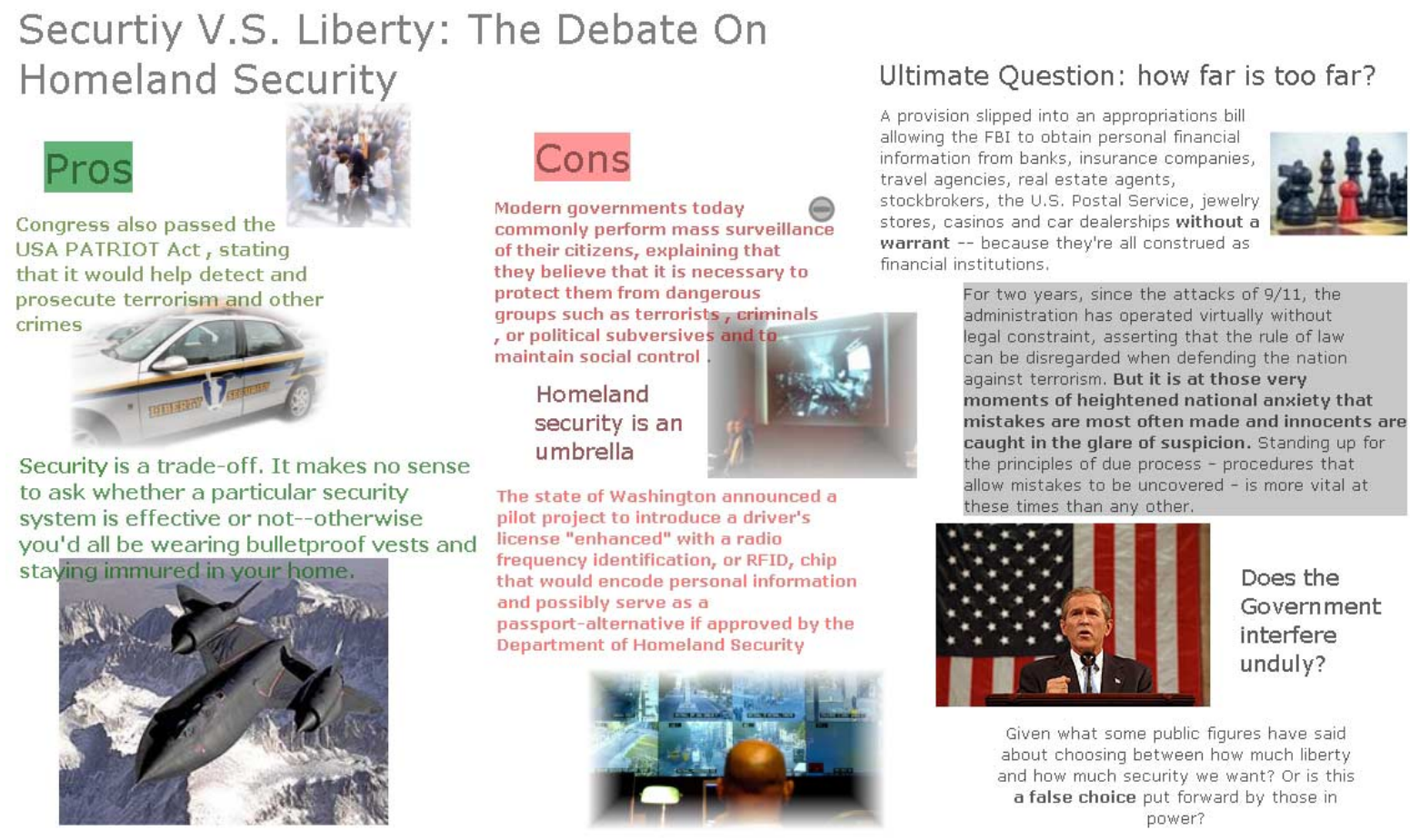

Figure 3: Composition created by participant with the integrated condition.

based ideation questions for training:

- "Compare and contrast urban and rural lifestyles. What is their impact on culture, politics, and quality of life?" Search queries were: (1) rural farm life, (2) skyscraper highrise architecture, (3) small town rural America, (4) traffic congestion, (5) urban amenities, and (6) urbanism.

- "Compare and contrast the ideas of development and ecology. Can we strike a balance between these two competing ideas? If so, how? " Search queries were: (1) deforestation expansion, (2) ecosystem conservation, (3) human impact on biodiversity, (4) land consumption for population growth, (5) sustainable ecology versus economic development, (6) sustainable land development.

The second session was conducted two days later. Subjects performed two more information-based ideation tasks:

- "Consider the possibilities of Stem Cell Research. Think of new ideas for how it can be used." Search queries were: (1) stem cell debate, (2) stem cell research, (3) miotic cell genetics cure embryonic, (4) cloned stem cell genes combat disease, (5) genetics ethics, and (6) regenerative cell DNA sequence.

- "Compare and contrast the ideas of liberty and security. Which is more important to you? How do the policies and actions of government impact these ideas?" Search queries were: (1) big brother government news, (2) liberty versus security, (3) homeland security, (4) freedoms lost to terrorism, (5) mass surveillance, and (6) civil liberties.

The following text appeared after all questions:

Express your ideas in an information composition. Articulate motivations and implications for yourself and society.

Develop and connect ideas, opinions, and explanations with regard to this line of research.

- Find and collect a variety of relevant information.

- Use your own ideas to create a coherent composition that develops original themes, strategies and directions.

- Use the composition to visualize relationships among information elements.

- Connect elements visually and conceptually.

- Develop a clear sense of what you are presenting and why.

- At the same time, let unexpected ideas emerge.

- Create concise annotations to articulate themes and explain what makes the collected elements important.

Use your time well. Remember to spend some of your time collecting relevant information, some organizing and designing, and some annotating with explanations.

Conditions and ideation tasks were counterbalanced. Each participant developed her answers to each information-based ideation question as an information composition (e.g. Figure 3).

The experiment concluded with the following set of postquestions, asking participants about their experiences:

1. Which version was more tedious to use?

2. Which version was more stimulating to use?

3 . Which version was easier to use?

4. Which version was more helpful to use?

5. Which version was more useful?

6. Which version was more fun to use?

7. Which version was easier to collect relevant information with?

8. Which version required less effort to use?

Permitted answers to these questions were: (a) separated, (b) integrated, and (c) both the same. 

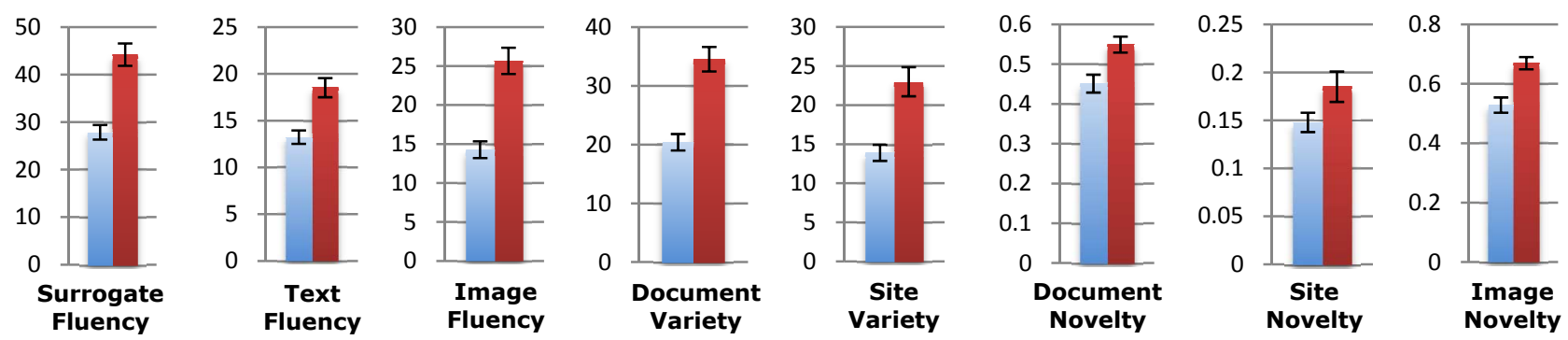

Figure 4: Means for eight information-based ideation metrics were computed to evaluate information composition products, comparing the separated control condition (in blue) with the integrated condition (in red). All significantly show that participants were more creative in the integrated condition. Error bars show standard errors of the mean.

\subsection{Results}

We extend the methodology of Shah et al to evaluate creativity of products developed by participants [32]. We apply computed metrics to evaluate the information compositions created by participants. We collected responses to post questions about user experiences comparing the two conditions.

\subsubsection{Computed Ideation Metrics}

We computed empirical formulations of Shah et al's fluency, variety, and novelty creative ideation metrics (Figure 4).

Fluency is the total number of generated ideas. We consider each surrogate the participant collects as representing an idea, and so measure the total number of surrogates collected. For the control condition, the average collection fluency was $27.88(\mathrm{SE}=1.54)$ surrogates, while for the integrated condition, the average was $44.2(\mathrm{SE}=2.36)$. The difference was significant, $t(82.6)=5.8, p<$ 0.0001 . We can separate the compositional fluency statistic into image and text components. The integrated authoring condition collected an average of 25.67 image surrogates versus 14.29 for the control, and again the results were significant, $t(81.2)=5.7, p$ $<0.0001$. The integrated condition collected $18.53(\mathrm{SE}=1.02)$ text surrogates versus $13.24(\mathrm{SE}=0.72)$ for the control; this difference is significant, $t(85.9)=4.2, p<0.0001$.

Variety measures the solution space explored during ideation. We presently measure variety as the diversity of web information that has been collected. We measured variety in terms of the number of documents and web sites that the participant collected information from. For source document variety, participants collected information from an average of $34.57(\mathrm{SE}=2.09)$ documents for the integrated condition, while the control condition average was $20.43(\mathrm{SE}=1.4)$ documents, and the difference was significant, $t(83.73)=5.6, p<0.0001$. For source web site statistics, site is based on top-level web domain (e.g. both money. cnn. com and weather.cnn.com reduce to the single domain cnn.com). The integrated authoring condition collected information from 23.0 source web sites, on average $(\mathrm{SE}=1.86)$, while the control condition averaged $13.9(\mathrm{SE}=1.03)$, and again the difference was significant, $t(74.9)=4.3, p<0.0001$.

Novelty measures how unusual an idea is, as compared to other ideas. We measure novelty in a manner analogous to the IDF measure of information retrieval [31]. We calculate element novelty scores $\operatorname{enov}_{t}(e)$ for each individual element $e$ of each type $t \epsilon$ image, document, site $\}$, over set of all compositions, $\boldsymbol{C}$.

$$
\operatorname{enov}_{t}(e)=\frac{1}{|c \in \boldsymbol{C}: e \epsilon c|}
$$

The more compositions that contain a particular image surrogate, the lower its novelty, enovimage $(e)$. Likewise, the more compositions that contain surrogates collected from a particular document or site, the less novel the source.

Then, to calculate compositional novelty scores $\operatorname{cnov}_{t}(c)$, for each type $t$ for each composition, we average the novelty scores for elements of type $t$ in that composition.

Mean compositional Image Novelty ( $\left.\overline{c n o v} \overline{\text { image }}_{\text {e }}\right)$ was significantly higher in the integrated condition than the control ( 0.67 vs. 0.53 ; $t(92)=4.28, p<0.0001)$. Mean Document Novelty $\left(\bar{c}_{\bar{c} o v_{\text {document }}}\right)$ for the integrated condition was also higher ( 0.19 vs. $0.15 ; t(82.5)$

$$
\operatorname{cnov}_{t}(c)=\frac{\sum_{e_{\text {of type } t: e \in c} \text { enov }_{t}(e)}}{\left|e_{\text {of type } t}: e \in c\right|}
$$

$=2.13, p=0.037)$. Mean Site Novelty $(\overline{c n o v}$ site $)$ was higher for the integrated condition $(0.185$ vs. $0.148 ; t(81.6)=1.98, p=0.05)$.

\subsubsection{Experience Reports}

Following completion of the two information-based ideation tasks for the second session, participants answered a set of post questions. These questions asked participants to compare experiences between the two conditions. We collected responses from 28 participants. Errors in the study apparatus prevented storing of responses from the other 21 participants. The results are presented in Figure 5. Responses were highly in favor of the integrated authoring condition.

A significant majority of participants said the integrated condition was less tedious $\left(\chi^{2}=15.5, \mathrm{p}=0.0005\right)$, easier to use $\left(\chi^{2}=30, \mathrm{p}<\right.$ $0.0001)$, more stimulating $\left(\chi^{2}=26, \mathrm{p}<0.0001\right)$, more helpful $\left(\chi^{2}=\right.$ $21.9, \mathrm{p}<0.0001)$, more fun to use $\left(\chi^{2}=38.9, \mathrm{p}<0.0001\right)$, more useful $\left(\chi^{2}=6.5, \mathrm{p}=0.00388\right)$, easier to collect relevant information $\left(\chi^{2}=12.3, \mathrm{p}=0.002\right)$, and required less effort to use $\left(\chi^{2}=44.85, \mathrm{p}<0.0001\right)$ than the control condition.

\subsubsection{Discussion}

Results from ideation metrics validate our hypothesis: integrating implicit structure visualization with authoring promotes ideation. Both conditions used MessyOrganizer for visualization and information composition for authoring, but integration varied. The novelty and variety metrics show that integration helps participants collect and think about different information. Separated visualization and authoring spaces make simultaneously thinking about information while exploring and collecting more challenging than an integrated environment.

Participants' experience reports demonstrate strong preference for the integrated condition. In some cases, participants were almost unanimous in this preference. Experience reports show that the integrated condition is easier to use, more stimulating, and requires less effort than separated spaces. Connecting reports on effort with computed metrics, we see an expected correlation 


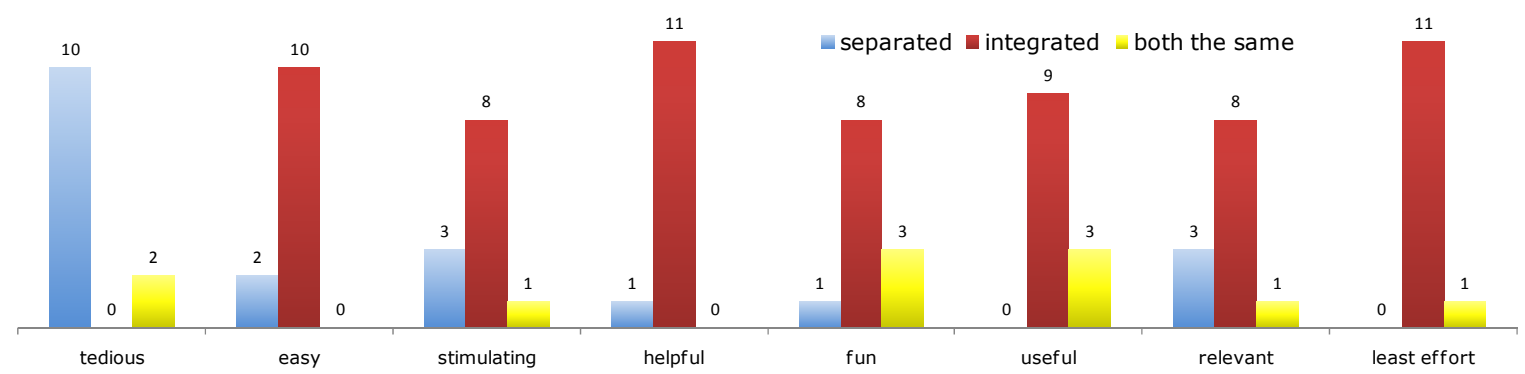

Figure 5: Participant responses comparing their experiences. The integrated condition was preferred by more participants for each aspect of their experiences. No participant found using the control condition to require less effort.

between reduced effort and creativity metrics. This indicates that commonly used drag and drop interaction across separate visualization and authoring windows imposes adverse effects on creative processes.

Previous studies found that information composition without visualization promoted creativity in information-based ideation tasks. These findings build upon the prior results emphasizing the benefit of supporting an integrated environment.

\section{CONCLUSION}

Incremental methods are required for visualization of collections that evolve over time. We developed a new incremental information visualization algorithm that conveys implicit relationships through spatial arrangement and supports integrated authoring. We evaluated effectiveness of integrating visualization with authoring in promoting information-based ideation. We found that participants developed more novel and varied ideas when visualization is integrated with authoring. Participant preferred an integrated environment for engaging in informationbased ideation tasks.

For intellectual work that requires developing creative ideas while working with information from a wide range of digital libraries and other information collections, we need new ways to support ideation. Implicit structure visualization has a significant role to play. We take an interdisciplinary approach, integrating cognitive psychology, the humanities, human-centered computing and visual design, to develop MessyOrganizer as evidence of this significance. The controlled experiment presented here builds on field studies, which showed that prior mixed-initiative information composition promotes creativity on assignments in an undergraduate class on invention and entrepreneurship [17][18].

We address an experience gap in information visualization. It is limiting to only consider explicit structure visualization. By extending the field to implicit structure visualization, information visualization can aid cognition for intellectual work, such as information-based ideation where visualizing content is necessary. Authoring processes, such as annotating and collecting, play a fundamental role. Implicit structure visualization integrated with authoring allows the user to simultaneously explore collections and think about information while also investigating new ideas. The hypothetical high school student Grace thinks about the information for her science project while collecting new information. The messiness of MessyOrganizer encourages her to meaningfully organize and author information. New ideas emerge. The implicit structure and messiness resulting thereof serve as a springboard to ideation. As a result, Grace develops many novel and varied ideas for her science project.

Integration extends beyond simply sharing a visual space.
Authoring and visualization spaces are connected by underlying semantics. Processes connected with visualization, such as searching, browsing, and retrieving, are directly related to processes associated with authoring, such as collecting and annotating. Semantics from information collected and authored can directly impact visualization, such as in MessyOrganizer, where cool space surrogates direct placement of new surrogates in the hot space. Metadata associated with surrogates also supports experiences of sensemaking. In Figure 1, the user looks at an image surrogate depicting a sketched design for a portable water purification device. However, what the image depicts is not immediately evident. Because semantics are maintained across the transition from visualization to authoring, the user is able to see metadata, including the image's caption, and the title, location, and categories of the source Wikipedia article. These semantics help users think about the relevance of the information represented by the surrogate and how it relates to other ideas she has collected.

In the experiences of creative tasks, like developing a new plan, project, or thesis, iterative processes of collecting and organizing information are inherently messy, because we do not know in advance exactly what information we will want, and what we will find significant in it. The messiness of MessyOrganizer's incremental visualization results directly from its simulation of and support for these human collecting processes. Alternate approaches for incremental visualization could achieve less messy solutions, but elements would require iterative spatial rearrangement, possibly with each new placement. The associated visual changes would be disruptive. Visual techniques such as animation could be employed to reduce the disruption, but working memory has limits. In future work, we will investigate alternate approaches to incremental visualizations and the effect of those visualizations on creativity. We will also investigate how implicit and explicit structure visualization can be complementary.

Implicit structure visualization integrated with authoring can play a key role in the value proposition of any digital library. Participants are supported in using information and in serendipitously discovering how the library's resources can be relevant to the task at hand, especially for information-based ideation tasks. Ideation is the foundation of innovation and invention across the humanities and sciences, in education, academia, and industry. We have presented strong evidence that integrating implicit structure visualization with authoring promotes ideation.

\section{REFERENCES}

[1] Abbasi, A., Chen, H. Categorization and analysis of text in computer mediated communication archives using visualization. Proc. JCDL 2007, 11-18.

[2] Ahern, S., Naaman, M., Nair, R., Yang, J.H. World explorer: 
visualizing aggregate data from unstructured text in georeferenced collections. Proc. JCDL, 2007, 1-10.

[3] Amar, R. A., Stasko, J. T. A knowledge task-based framework for design and evaluation of information visualizations. IEEE InfoVis 2004, 143-150.

[4] Bertini, E., Plaisant, C., Santucci, G., Beliv '06: Proceedings of the 2006 AVI workshop on BEyond time and errors.

[5] Burke, M., Organization of Multimedia Resources, Hampshire, UK: Gower, 1999.

[6] Butcher, K.R., Bhushan, S., Sumner, T. Multimedia displays for conceptual discovery: information seeking with strand maps. Multimedia Systems 11, 3 (2006), 236-248.

[7] Card, S. K., Mackinlay, J. D., Shneiderman, B. Readings in Information Visualization: Using Vision to Think. Academic Press, London, 1999.

[8] Card, S. K., Robertson, G. G., York, W. The webbook and the web forager: an information workspace for the worldwide web. $C H I$ '96, 111-117.

[9] Conklin, J., Begeman, M.L. gIBIS: a hypertext tool for exploratory policy discussion. ACM TOIS 6:4 (10/1988).

[10] Druin, A., Bederson, B.B., Hourcade, J.P., Sherman, L., Revelle, G., Platner, M., Weng, S. Designing a digital library for young children. Proc. JCDL 2001, 398-405.

[11] Fass, A., Forlizzi, J., Pausch, R. MessyDesk and MessyBoard: two designs inspired by the goal of improving human memory. Proc. DIS 2002, 303-311.

[12] Finke, R. A., Ward, T. B., Smith, S. M. Creative Cognition. MIT Press, Cambridge, MA, 1992.

[13] Flickr: "hurricanekatrina", last visited Jan. 29, 2011, http://www.flickr.com/photos/tags/hurricanekatrina/.

[14] Fu, X., Ciszek, T., Marchionini, G., Solomon, P. Annotating the Web: An Exploratory Study of Web Users' Needs for Personal Annotation Tools. Proc. ASIST 2005, 42.

[15] Greene, S., Marchionini, G., Plaisant, C., Shneiderman, B. Previews and Overviews in Digital Libraries: Designing Surrogates to Support Visual Information Seeking. Journal of the American Society for Info. Science 51, 4 (2000), 380-393.

[16] Kang, H., Plaisant, C., Lee, B., Bederson, B.B. NetLens: iterative exploration of content-actor network data. Information Visualization 6, 1 (March 2007), 18-31.

[17] Kerne, A., Koh, E. Representing Collections as Compositions to Support Distributed Creative Cognition and Situated Creative Learning, New Review of Hypermedia and Multimedia (NRHM), 13(2) Dec 2007, 135-162.

[18] Kerne, A., Koh, E., Smith, S.M., Webb, A., Dworaczyk, B. combinformation: Mixed-initiative composition of image and text surrogates promotes information discovery. ACM Trans. Info. Syst. 27, 1 (2008), 1-45.

[19] Kerne, A., Smith, S.M. The information discovery framework. Proc DIS 2004, 357-360.

[20] Kerne, A., Smith, S. M., Koh, E., Choi, H., Graeber, R. An experimental method for measuring the emergence of new ideas in information discovery. IJHCI 24, 5 (2008), 460-477.

[21] Kirkpatrick, S., Gelatt, C.D., Vecchi, M.P. Optimization by simulated annealing. Science 220 (1983), 671-680.

[22] Marchionini, G. Exploratory search: from finding to understanding. $C A C M, 49,4$ (April 2006), 41-46.

[23] Marshall, C.C. Annotation: from paper books to the digital library. Proc. Digital Libraries 1997, 131-140.

[24] Marshall, C.C. Bly, S. Saving and using encountered information: implications for electronic periodicals. Proc. CHI, 2005, 111-120.

[25] Marshall, C.C., Rogers, R.A.. Two years before the mist: experiences with Aquanet. In Proc. Hypertext 1992, 53-62.

[26] Marshall, C.C., Shipman, F.M. Spatial hypertext: designing for change. CACM 38, 8 (August 1995).

[27] O'Hara, K., Sellen, A. A comparison of reading paper and on-line documents. Proc. CHI 1998, 335-342.

[28] Pirolli, P., Card, S. K. Information foraging. Psychological Review 106 (1999), 643-675.

[29] Robertson, G., Czerwinski, M., Larson, K., Robbins, D. C., Thiel, D., van Dantzich, M. Data mountain: using spatial memory for document management. UIST '98, 153-162.

[30] Russell, D., Stefik, M.J., Pirolli, P., Card, S.K. The cost structure of sensemaking. Proc.CHI, 1993, 269-276.

[31] Salton, G., McGill, M. J. Introduction to Modern Information Retrieval. McGraw-Hill, New York, 1986.

[32] Shah, J.J., Smith, S.M., Vargas-Hernandez, N. Metrics for Measuring Ideation Effectiveness, Design Studies, 24:2 (2003), 111-134

[33] Shen, R., Vemuri, N.S., Fan, W., Torres, R da S., Fox, E.A. Exploring digital libraries: integrating browsing, searching, and visualization. Proc. JCDL, 2006, 1-10.

[34] Shipman, F.M., Marshall, C.C. Formality Considered Harmful: Experiences, Emerging Themes, and Directions on the Use of Formal Representations, in Interactive Systems. Comput. Support. Coop. Work 8, 4 (October 1999), 333-352.

[35] Simon, H. A. How Big Is a Chunk? Science 183 (Feb. 1974).

[36] Simonton, D.K. Creativity as Blind Variation and Selective Retention: Is the Creative Process Darwinian? Psychological Inquiry 10, 4 (1999), 309-328.

[37] Smith, M.A., Shneiderman, B., Milic-Frayling, N., Rodrigues, E.M., Barash, V., Dunne, C., Capone, T., Perer, A., Gleave, E. Analyzing (social media) networks with NodeXL. In Proc.Communities and Tech. 2009, 255-264.

[38] Smith, S. Fixation, incubation, and insight in memory and creative thinking. In The Creative Cognitive Approach, (Smith, S., et al., Eds.), MIT Press, 135-156.

[39] Tufte, E. R. Envisioning Information, 4th printing ed. Graphics Press, May 1990.

[40] Viegas, F.B., Wattenberg, M., van Ham, F., Kriss, J., McKeon, M. ManyEyes: a Site for Visualization at Internet Scale. IEEE Trans. Vis. \& Comp. Graphics 13, 6 (Nov 2007).

[41] Webb, A., Kerne, A. The in-context slider: a fluid interface component for visualization and adjustment of values while authoring. Proc. AVI, 2008, 91-99.

[42] Wildemuth, B.M., Marchionini, G., Yang, M., Geisler, G., Wilkens, T., Hughes, A., Gruss, R. How fast is too fast?: evaluating fast forward surrogates for digital video. Proc. JCDL, 2003, 221-230.

[43] Xu, W., Esteva, M., Jain, S.D. Visualizing personal digital collections. Proc. JCDL, 2010, 169-172. 